\title{
The imperative to prevent diabetes complications: a broadening spectrum and an increasing burden despite improved outcomes
}

the opportunity currently exists

to prevent onset of complications

in most patients

Stephen $\mathbf{M}$ Twigg MBBS(Hons), PhD, FRACP, 12

Jencia Wong MBChB, PhD, FRACP 1.2

1 University of Sydney, Sydney, NSW.

2 Royal Prince Alfred Hospital Sydney, NSW.

stephen.twigg@ sydney.edu.au

doi: 10.5694/mjal4.01234
W hile the diabetes epidemic continues to gather pace globally, there is cause for both optimism and concern with regard to diabetes complications. A recent Australian study, in which data from a national diabetes registry collected from 1997 to 2010 was interrogated, showed that mortality rates in diabetes are decreasing, ${ }^{1}$ in line with secular decreases seen globally. Recent overseas data also show that life expectancy in type 1 diabetes is improving, yet the risk of death from any cause or from cardiovascular causes remains at least twofold higher for such patients compared with the general population. ${ }^{2}$ Thus, mortality outcomes are improving in people with diabetes and, overall, are better aligning with those of the general population. For example, Australian data indicate that between 1997 and 2010, the diabetes-related death rate for all Australians declined by $20 \%{ }^{3}$

These data, however, relate particularly to older adults, who generally now lose very few years of life due to diabetes. In contrast, in younger adults with type 1 and type 2 diabetes, mortality has remained high compared with age-matched controls. ${ }^{1,4}$ Further, subgroups including Indigenous Australians, some people born overseas and those living outside major cities of Australia have notably higher diabetes-related death rates. ${ }^{3}$ The data on complications suggest that improved strategies should now be directed to these subgroups to reduce the withindiabetes differential in mortality outcomes.

Superimposed on the general decreases in mortality rates are indications that the rates of the more severe complications in people with diabetes are also declining. Recent analysis of large US databases has shown that rates of myocardial infarct, stroke and amputation are falling. ${ }^{5}$ These secular improvements may in part be due to the earlier detection of type 2 diabetes in recent years, resulting in a less severe patient phenotype being studied - a form of lead-time bias. Nevertheless, a major factor has been the improved treatment of people with diabetes, especially in terms of modifiable risk factors; improvements in lipid levels, blood pressure and blood glucose levels and reduced background rates of smoking have each likely played a role. ${ }^{1}$ Screening and detecting diabetes complications, by history-taking and clinical examination plus dedicated screening for vascular complications, may have also contributed to improved outcomes.

Such optimism, however, gives way to concern; the combination of improvements in survival in those with diabetes and an increase in new diabetes diagnoses (related
Summary

- Diabetes mellitus and its complications are common; the complications are, of themselves, a major reason to manage diabetes.

- Recent data from Australia and similar developed health care systems overseas indicate that morbidity and mortality outcomes relating to diabetes complications are improving.

- However, these benefits are offset by increasing numbers of people diagnosed with diabetes, resulting in an increased disease burden with significant health care implications. Thus the imperative to prevent diabetes and diabetes complications has never been greater.

- Furthermore, the recognised spectrum of diabetes complications is broadening, especially complications relating to lipid levels, insulin resistance and the metabolic syndrome. Clinicians now need to be aware of both traditional complications (eg, nephropathy and cardiovascular disease) and non-traditional complications (eg, polycystic ovary syndrome, non-alcoholic fatty liver disease, some cancers and eating disorders).

- Complications outcomes could be further improved by decreasing the evidence-treatment gap - for example, by increasing personalisation of care in managing diabetes complications.

to unchecked obesity and an ageing population) are contributing to increased diabetes prevalence and disease burden. ${ }^{6}$ Thus, although the incidence of complications is declining, these benefits are overwhelmed by the sheer numbers of people affected by diabetes. This includes increasing projected numbers of younger patients with diabetes who have a high lifetime risk of complications. ${ }^{7}$ With current trends, the community burden of diabetes complications is set to increase. Therefore, a net result of our treatment success is an increase in current and projected population disability in people with diabetes (Appendix 1). ${ }^{8}$ Disability-adjusted life-years (DALYs) are projected to rise for diabetes; it has been predicted that diabetes will have the highest national disability score for any chronic illness from $2016 .{ }^{8}$ In addition, there are increasing direct health and resource costs attributed to diabetes, estimated at $\$ 1.5$ billion for the 2008-09 financial year, which represents an $86 \%$ increase over an 8-year period. ${ }^{9}$ With this increasing burden, the challenge has never been greater to prevent diabetes and related complications. 
Epidemiology of diabetes complications in Australia: clinical implications

Despite recent advances, complications of diabetes are a significant cause of morbidity and mortality. Diabetes is the most common cause of end-stage renal failure and of working-age blindness in Australia. However, it is notable that at any time point, most people with diabetes do not have any diabetes complications (Box 1$).{ }^{10}$ About one-third will have one or two complications, and only a minority will have more than two. The proportion of people with a severe complication is much lower - less than $5 \%$ each year for conditions including myocardial infarct, blindness, end-stage renal disease and severe hypoglycaemia.

These data inform us that, for most patients, we will be attempting to prevent onset of diabetes complications, and screening for their occurrence to better target and intensify therapy for complications that are detected. This is an important message; the opportunity currently exists to prevent onset of complications in most patients. In other patients, preventing the worsening of complications will be the aim. In those with severe endstage complications, and in the absence of possible organ transplantation, the focus will be on palliation and active management of adverse symptoms caused by diabetes and its management.

\section{A constellation of comorbidities: the broadening complications spectrum}

To date, prevention and management of diabetes complications have focused on the long-term end-organ blood glucose-related microvascular complications (nephropathy, retinopathy and neuropathy) and macrovascular complications (cardiovascular disease [CVD], cerebrovascular disease and peripheral vascular disease), and on foot disease, as these are major causes of morbidity and premature mortality. However, the spectrum of diabetes complications is not restricted to these "traditional" forms.

The acute complications of diabetes, such as diabetic ketoacidosis or hyperosmolar coma, are part of the complications spectrum. Increasingly, "non-traditional" diabetes-related complications are being recognised, given their frequent co-occurrence with and in some cases their exacerbation by diabetes, as well as their impact on outcomes for individuals with diabetes. These include complications related to insulin resistance and the metabolic syndrome, including obstructive sleep apnoea, polycystic ovary syndrome, gout, the common diastolic dysfunction in diabetic cardiomyopathy, and non-alcoholic fatty liver disease (NAFLD). Indeed, NAFLD is more common in people with type 2 diabetes, and diabetes accelerates NAFLD to its more severe form of non-alcoholic steatohepatitis. ${ }^{11}$

Certain infections and some cancers (eg, colorectal, breast, liver and pancreas cancers) are linked to the metabolic syndrome, and cancer is an increasingly prevalent cause
1 Prevalence and incidence of diabetes complications (type 1 and type 2 diabetes combined), according to a National Association of Diabetes Centres audit and benchmarking report, $2011^{10}$

Proportion

Prevalence of traditional complications (any severity)

0

$61.7 \%$

1 or 2

$30.0 \%$

3 or more

$8.3 \%$

Incidence of severe complications in previous 12 months

Myocardial infarct

$4.0 \%$

Coronary artery bypass grafting, angioplasty or stent

$2.7 \%$

Stroke

$1.6 \%$

New blindness

$0.9 \%$

End-stage renal disease

$0.4 \%$

Lower limb amputation

$0.7 \%$

Severe hypoglycaemia

of death in patients with diabetes. ${ }^{1}$ Given increased longevity, diabetes-related cognitive impairment leading to dementia is becoming a clinically significant problem for our ageing population, ${ }^{12}$ as is heart failure. Periodontal disease ${ }^{13}$ and low testosterone in men with type 2 diabetes $^{14}$ are also prevalent but less well recognised. In people with type 1 diabetes, related autoimmune diseases require regular consideration in care; these include thyroid disease (Hashimoto disease and Graves disease), coeliac disease, premature ovarian failure and Addison disease. ${ }^{15}$

Further, in considering the "biopsychosocial model", prevalence of major mood state disorder (especially clinical depression) is elevated in people with diabetes compared with the general population. ${ }^{16}$ Also, forms of eating disorders are clearly increased in type 1 diabetes, ${ }^{17}$ as is the diabetes-specific psychological condition of "diabetesrelated distress", which links to the emotional burden, and demands in regimen, of having diabetes. Clinicians should be aware of such complications and comorbidities in patients and that management may fall beyond traditional diabetes paradigms. In addition, these are variably included in health economic analyses and, if accounted for, health costs of complications are likely to be greater.

\section{Clinical lessons from studies of the pathogenesis of diabetes complications}

The pathogenesis of tissue and organ diabetes complications is challenging to study as clinical disease usually takes many years to develop and progress. In addition, the propensity to develop diabetes complications is not entirely predictable from clinical profiles and risk factors alone. Genomic risk profiling in diabetes complications is not yet a clinical tool. Nevertheless, clinicians should be alert to family histories of complications as a clue to enhanced complications susceptibility, particularly for diabetic renal disease. ${ }^{18}$ 
Progress has been made in defining how chronic high blood glucose levels can, via cell and tissue intermediates such as advanced glycation end-products or growth factors, lead to inflammation and/or fibrosis in an organ and subsequent loss of function. ${ }^{19}$ In addition, there has been some success in targeting intermediates downstream of glycaemia to attenuate organ complications. ${ }^{20}$ For example, fenofibrate has been shown to have clinical utility in preventing some diabetes complications, most notably the progression of diabetic retinopathy, ${ }^{21}$ probably through mechanisms including antioxidant and peroxisome proliferator-activated receptor- $\alpha$ modulating effects. Further, in patients with severe vision threatening retinopathy, blockade of vascular endothelial growth factor (VEGF) bioactivity through intraocular antibody strategies can not only prevent blindness in those with diabetic macular oedema but also improve visual acuity. ${ }^{22}$ These findings show that if blood glucose control has not prevented severe diabetes complications in a particular patient, other clinical interventions might salvage a threatened organ.

Prevention of diabetes complications: glucose in perspective

\section{The evolving glucocentric approach}

Only late last century through randomised controlled trials targeting glycaemia was it shown that metabolic control matters in diabetes complications. In type 1 and type 2 diabetes, prevention of onset and progression of microvascular complications of diabetes was realised by chronic control of blood glucose levels. ${ }^{23,24}$ Subsequently, the epidemiological follow-up cohorts of the Diabetes Control and Complications Trial (DCCT) ${ }^{25}$ and the UK Prospective Diabetes Study (UKPDS) ${ }^{26}$ showed that macrovascular complications can be prevented by tight chronic glycaemic control in type 1 and type 2 diabetes, respectively. These studies also showed that early glucose control delivers sustained benefit in reducing the risk of onset and progression of diabetes complications across subsequent years and decades. From these observations, the concept of "metabolic memory" or "legacy effect" of early blood glucose control arose. ${ }^{25,26}$

Subsequent randomised controlled trials of chronic glycaemia - such as the Action in Diabetes and Vascular Disease: Preterax and Diamicron Modified Release Controlled Evaluation (ADVANCE), ${ }^{27}$ the Veterans Affairs Diabetes Trial (VADT) ${ }^{28}$ and the Action to Control Cardiovascular Risk in Diabetes (ACCORD) trial ${ }^{29}$ have collectively shown that microvascular complications can also be prevented by tight chronic glycaemic control. These studies enrolled patients with more advanced diabetes than the DCCT and the UKPDS and did not show benefit in macrovascular disease or mortality. In addition, the ACCORD trial showed that harm as well as good can occur when a therapeutic strategy to intensively target blood glucose levels is used in patients with more advanced disease. ${ }^{29}$ Although the causality has not been defined for the ACCORD trial findings, together these studies of chronic glycaemia have led to the concept of individualisation of blood glucose targets to improve the benefit-to-risk ratio in people with diabetes. ${ }^{30}$

Specific regulation of postprandial glucose and its importance in terms of risk of complications in diabetes is unclear, according to a recent review by the International Diabetes Federation. ${ }^{31}$ The observational Diabetes Epidemiology: Collaborative Analysis of Diagnostic Criteria in Europe (DECODE) study showed a relationship between postprandial dysglycaemia and risk of $\mathrm{CVD}^{32}$ while the Study to Prevent NIDDM (STOPNIDDM Trial), which used acarbose to target postprandial dysglycaemia, showed benefit in terms of CVD but was underpowered to do so. ${ }^{33}$ Although it has not yet been shown whether targeting postprandial glucose has a benefit in terms of complications prevention above glycated haemoglobin $\left(\mathrm{HbA}_{1 c}\right)$ reduction alone, achieving an $\mathrm{HbA}_{1 \mathrm{c}}$ level of $\leqslant 53 \mathrm{mmol} / \mathrm{mol}$ can only be safely realised in a patient by considering postprandial glucose levels and fasting levels.

\section{Challenges in maintaining chronic glycaemic control}

The decline in $\beta$-cell function that is usual in type 2 diabetes, and the increasing risk of hypoglycaemia in type 1 diabetes over time or after antecedent hypoglycaemia, antagonise the goal of durable glycaemic control. For type 1 diabetes, the hypoglycaemia risk is especially increased and related to autonomic dysfunction affecting the release of counter-regulatory hormones (glucagon and adrenalin). If insulin therapy cannot be further optimised, a higher $\mathrm{HbA}_{1 c}$ target may be required with the unavoidable compromise being a higher risk of complications. For type 2 diabetes, the need for clinicians to continue to overcome therapeutic inertia in the intensification of treatment is critical; in particular, clinicians must impress on patients the usual need for titrated pharmacotherapy in addition to lifestyle interventions.

Major challenges that add complexity to care for people with diabetes include new medicines and combinations. Expansion of treatments on the Pharmaceutical Benefits Scheme and under the Therapeutic Goods Administration requires that health professionals are continually upskilled through evidence-based education in the use of medicines for diabetes. Similarly, it remains to be seen whether the newer agents available for type 2 diabetes, including therapies based on incretin and sodium-glucose cotransporter-2 - which show varying but largely beneficial effects on non-glycaemic risk profiles in preliminary studies, including body weight and blood pressure reductions - will translate into benefits relating to complications in clinical trials. Data from cardiovascular trials involving newer hypoglycaemic agents that are currently underway, and postmarketing data for these agents, should shed light on this.

\section{The multifactorial approach}

In parallel with the trials examining glycaemia are key clinical trials such as the Collaborative Atorvastatin 


\section{Strategies and methods to prevent and treat diabetes complications*}

Complications strategy, and parameter or complication

Diagnose diabetes in a timely manner

Engage the patient with diabetes in self-care

Intensively target and manage major reversible multifactorial risk factors for organ complications*

Chronic glycaemia

Lipid levels

Blood pressure

Smoking cessation

Sustained weight loss if indicated

CVD absolute risk

Screen for traditional complications, generally at least annually

Eyes

Kidneys

Peripheral nerves, including feet

Cardiovascular

Cerebrovascular

Peripheral arterial

Once a particular complication is detected, consider: increased frequency of specific complication reassessment; specific endorgan therapy for the complication, including antiplatelet and anticoagulant strategies in macrovascular disease; specialist referral

Retinopathy

Nephropathy

Neuropathy

Ischaemic heart disease

Transient ischaemic attack or stroke

Peripheral arterial disease

\section{Consider presence of other complicationsફ}

Depression, diabetes distress

Diabetic gastroparesis

Erectile dysfunction

Establish a hypoglycaemia prevention and therapy plan

(especially for those receiving insulin therapy)

Educate in hypoglycaemia prevention and treatment

Assess for lack of hypoglycaemia awareness, severe hypoglycaemi history

Establish a sick day management plan (for hyperosmolar non-

ketotic coma or diabetic ketoacidosis)

Self-care education during intercurrent illness
Summarised intervention or assessment methods

Use current clinical care guidelines to screen for type 2 diabetes, including glycated haemoglobin level where indicated (now approved on the Medicare Benefits Schedule)

Develop a rapport and use chronic care models to aid self-care adherence; consider e-health initiatives (eg, medical appointment reminders)

Glycated haemoglobin generic target: $\leqslant 53 \mathrm{mmol} / \mathrm{mol}^{\dagger}$

LDL-C generic target: $<2.0 \mathrm{mmol} / \mathrm{L}^{\dagger}$

Blood pressure generic target: $\leqslant 130 / 80 \mathrm{mmHg}^{\dagger \dagger}$

Include quit smoking programs

Calorie-controlled nutrition approach and focus on exercise

Consider reduction in absolute CVD risk (eg, statin therapy is recommended for patients at high risk of CVD irrespective of LDL-C level unless contraindicated or clinically inappropriate)

Perform dilated fundus examination and test visual acuity

Test for albuminuria and estimated glomerular filtration rate

Clinically assess by using a $10 \mathrm{~g}$ monofilament, testing reflexes, applying a tuning fork, and looking for skin and mechanical foot abnormalities

Clinically assess, especially by history-taking

Clinically assess (eg, check for carotid bruit)

Clinically assess (eg, check foot pulses)

Consider fenofibrate therapy in patients with type 2 diabetes and ophthalmologist referral for those with mild or higher grade of retinopathy

Commence angiotensin-converting-enzyme inhibitor or angiotensin receptor blocker therapy as tolerated in patients with albuminuria; consider nephrologist referral for patients with estimated glomerular filtration rate $<30 \mathrm{~mL} / \mathrm{min} / 1.73 \mathrm{~m}^{2}$ and those with macroalbuminuria

Refer patients with moderate or high risk of foot ulcer to a podiatrist for education and pressure offloading; ulcers should be managed with high-risk foot service care Refer patients with evidence of ischaemic heart disease to a cardiologist and stratify patients by urgency of review

Refer at-risk patients to a neurologist; assess by carotid duplex ultrasound

Consider referring patients to a vascular surgeon, especially those with claudication or rest pain

Assess by administering the Patient Health Questionnaire-2 or Problem Areas in Diabetes Questionnaire

Assess by history-taking; perform gastric-emptying study or a generic trial of prokinetic agents

Assess by history-taking; trial medical therapy; consider surgical interventions

Provide advice on balancing carbohydrate intake, exercise and insulin doses, and on frequency and timing of blood glucose monitoring; provide a specific hypoglycaemia self-treatment plan and an emergency plan

Assess by history-taking; if present, consider referring patients to an endocrinologist, diabetes educator or diabetes centre

Provide advice on frequency of self-monitoring of blood glucose and ketones, oral intake and insulin doses; provide an emergency plan

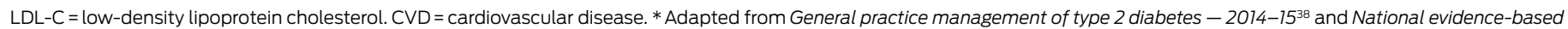

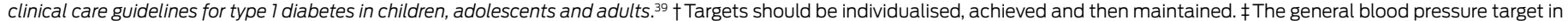
these guidelines remains $\leqslant 130 / 80 \mathrm{mmHg}$ despite it being relaxed in some recent guidelines. $\$$ Common examples only are given. 
Diabetes Study (CARDS), ${ }^{34}$ Heart Protection Study (HPS), ${ }^{35}$ the Ongoing Telmisartan Alone and in Combination with Ramipril Global Endpoint Trial (ONTARGET) and the Telmisartan Randomised Assessment Study in ACE Intolerant Subjects with Cardiovascular Disease (TRANSCEND), ${ }^{36}$ which have shown the importance of managing key lipid parameters and blood pressure in curtailing diabetes complications. In contrast to glycaemia, the achievement of blood pressure and lipid targets remain generally stable over time. The Steno-2 Study and follow-up study showed the significant benefit of a multifactorial approach targeting all CVD risk factors in concert, especially in people with albuminuria. ${ }^{37}$ The results of these studies emphasised that the relative impact of glycaemic control on macrovascular disease is quite modest, and that a major multifactorial emphasis on managing blood pressure, lipid levels and smoking cessation is essential in type 1 and type 2 diabetes.

\section{Outcomes in complications screening and risk factor management}

Practical strategies and related methods to aid complications outcomes in diabetes are provided in Box 2. ${ }^{38,39}$ Systematic practice of such evidence-based medicine will lead to improved patient outcomes. As reflected in recent data, the major concern is the gap between current evidence using available therapies and delivery of evidence-based care (although the gap is decreasing) ${ }^{40}$ According to national data, most people with diabetes continue to not receive the complete annual cycle of care for diabetes complications screening and many are not achieving the targets (albeit generic) established in national evidence-based diabetes clinical care guidelines for blood glucose levels, blood pressure and lipid levels (Appendix 2). ${ }^{10}$ It is expected that through improved national data capture processes and diabetes complications screening programs, documented outcomes regarding managing these reversible risk factors and the related diabetes complications will also improve. ${ }^{40,41}$ Each clinical practice needs to have systematised approaches to diabetes care, and each patient needs to have his or her care personalised in goal setting and delivery to optimise diabetes complications outcomes.

Clinicians need to know the current best clinical evidence to practise and adapt for each patient. The increased availability of user-friendly evidence-based clinical care guidelines in diabetes and complications in both type 1 and type 2 diabetes across the lifecycle facilitates this process. ${ }^{39,42}$ Increasingly, these guidelines acknowledge that a generic approach has value, although a one-size-fits-all approach does not work for many patients and health care delivery settings. Thus, quality clinical care guidelines developed by the National Health and Medical Research Council include clinical practice points to help personalise care by adapting the evidence to individual patients with diabetes. Examples include type 1 diabetes, paediatric diabetes and complicated type 2 diabetes (eg, diabetic
3 Key learning points

- By applying current readily available evidence-based knowledge to individual patients with diabetes, much can be done to prevent onset and progression of diabetes complications.

- Primary care physicians have a major role in diagnosing diabetes in a timely manner and then intensively managing risk factors for diabetes complications.

- Clinicians need to recognise the import of early glucose control, and an individualised and targeted multidisciplinary approach, in complications prevention. Focusing exclusively on glucose levels is insufficient to attenuate risk; a multifactorial approach with attention to cardiovascular risk factors is optimal.

- Detecting diabetes complications by systematic screening can aid targeting of therapies to improve outcomes relating to complications.

- Engaging patients in self-care, including through a diabetes health care team and e-health, can improve adherence to therapy and outcomes.

- In the future, it is envisaged that more sensitive markers for detecting complications subclinically and options to screen for a broader range of diabetes complications, combined with improved tissue-specific therapies, will further improve complications outcomes.

foot ulceration), which are each best managed directly by a multidisciplinary care specialist team including an endocrinologist or diabetologist, diabetes educator and nutritionist. In contrast, most patients with type 2 diabetes can be managed at a local and primary health care team level, with request for timely specialist consultation only in specific cases.

Obstetric care in diabetes requires a separate strategy that involves preconception planning (in patients with preexisting diabetes) and high-risk pregnancy care (including in patients with gestational diabetes). This topic is beyond the scope of this article.

\section{Diabetes complications now and into the future}

As understanding of diabetes complications improves, approaches to not only prevent onset and progression but to reverse complications and loss of organ function will become increasingly possible. Some potential initiatives are addressed in Appendix 3. In the interim, improved trends in clinical outcomes tell us that there is much to be achieved by systematically applying the current evidence base and clinical tools to prevent and manage complications for the increasing number of people affected by diabetes (Box 3).

Competing interests: Stephen Twigg is on advisory panels for Abbott Diabetes Care, AstraZeneca, Novo Nordisk, Sanofi, Takeda; has received research support from Novo Nordisk; and payment for service on speakers bureaus from AstraZeneca, MSD, Novartis, Novo Nordisk, Sanofi, Servier and Takeda. Jencia Wong and the institutions with which she is affiliated have received research funds, travel grants and speaker honoraria from various companies including Eli Lilly and Company, Boehringer Ingelheim, Novo Nordisk, MSD, AstraZeneca, Bristol-Myers Squibb, Novartis, Sanofi and Servier.

Provenance: Commissioned; externally peer reviewed.

References are available online at www.mja.com.au. 
1 Harding JL, Shaw JE, Peeters A, et al. Mortality trends among people with type 1 and type 2 diabetes in Australia: 1997-2010. Diabetes Care 2014; 37: 2579-2586.

2 Lind M, Svensson AM, Kosiborod M, et al. Glycemic control and excess mortality in type 1 diabetes. NEngl J Med 2014; 371: 1972-1982.

3 Australian Institute of Health and Welfare. Diabetes deaths. Canberra: AlHW, 2010. http://www.aihw.gov.au/diabetesindicators/deaths (accessed Dec 2014).

4 Constantino MI, Molyneaux L, Limacher-Gisler F, et al. Longterm complications and mortality in young-onset diabetes: type 2 diabetes is more hazardous and lethal than type 1 diabetes. Diabetes Care 2013; 36: 3863-3869.

5 Gregg EW, Li Y, Wang J, et al. Changes in diabetes-related complications in the United States, 1990-2010. N Engl J Med 2014; 370: 1514-1523.

6 Guariguata L, Whiting DR, Hambleton I, et al. Global estimates of diabetes prevalence for 2013 and projections for 2035. Diabetes Res Clin Pract 2014; 103: 137-149.

7 Imperatore G, Boyle JP, Thompson TJ, et al. Projections of type 1 and type 2 diabetes burden in the US population aged $<20$ years through 2050: dynamic modeling of incidence, mortality, and population growth. Diabetes Care 2012; 35: 2515-2520.

8 Australian Institute of Health and Welfare. Diabetes and disability: impairments, activity limitations, participation restrictions and comorbidities. Canberra: AlHW, 2013. (AlHW Cat. No. CVD 63; Diabetes Series No. 20.) http://www.aihw. gov.au/WorkArea/DownloadAsset.aspx?id=60129544708 (accessed Feb 2015).

9 Australian Institute of Health and Welfare. Type 2 diabetes in Australia's children and young people: a working paper. Canberra: AlHW, 2014. Canberra: (AlHW Cat. No. CVD 64; Diabetes Series No. 21.) http://www.aihw.gov.au/WorkArea/ DownloadAsset.aspx?id=60129546359 (accessed Mar 2015).

10 National Association of Diabetes Centres. Final report: Australian National Diabetes Information Audit and Benchmarking, December 2011. Canberra: Australian Government Department of Health, 2011. http://www.health. gov.au/internet/publications/publishing.nsf/Content/ pq-diabetes-pubs-andiabll-toc/\$FILE/ANDIAB-FinalReport-2011.pdf (accessed Feb 2015).

1 Williams KH, Shackel NA, Gorrell MD, et al. Diabetes and nonalcoholic fatty liver disease: a pathogenic duo. Endocr Rev 2013; 34: 84-129.

12 Ninomiya T. Diabetes mellitus and dementia. Curr Diab Rep 2014; 14: 487.

13 Bascones-Martínez A, González-Febles J, Sanz-Esporrín J. Diabetes and periodontal disease. Review of the literature. Am J Dent 2014; 27: 63-67.

14 Grossmann M. Low testosterone in men with type 2 diabetes: significance and treatment. J Clin Endocrinol Metab 2011; 96 : 2341-2353.

15 De Block CE, De Leeuw IH, Vertommen JJ, et al. Beta-cell, thyroid, gastric, adrenal and coeliac autoimmunity and HLA-DQ types in type 1 diabetes. Clin Exp Immunol 2001; 126: 236-241.

16 Hasan SS, Mamun AA, Clavarino AM, Kairuz T. Incidence and risk of depression associated with diabetes in adults: evidence from longitudinal studies. Community Ment Health J 2015; 51: 204-210.
17 Williams G, Gill GV. Eating disorders and diabetic complications [editorial]. N Engl J Med 1997; 336: 1905-1906.

18 Freedman BI, Bostrom M, Daeihagh P, et al. Genetic factors in diabetic nephropathy. Clin J Am Soc Nephrol 2007; 2: 1306-1316.

19 Forbes JM, Cooper ME. Mechanisms of diabetic complications. Physiol Rev 2013; 93: 137-188.

20 Nishikawa T, Edelstein D, Brownlee M. The missing link: a single unifying mechanism for diabetic complications. Kidney Int Suppl 2000; 77: S26-S30.

21 Simó R, Roy S, Behar-Cohen F, et al. Fenofibrate: a new treatment for diabetic retinopathy. Molecular mechanisms and future perspectives. Curr Med Chem 2013; 20: 3258-3266.

22 Titchenell PM, Antonetti DA. Using the past to inform the future: anti-VEGF therapy as a road map to develop novel therapies for diabetic retinopathy. Diabetes 2013; 62: 1808-1815.

23 Nathan DM. The pathophysiology of diabetic complications: how much does the glucose hypothesis explain? Ann Intern Med 1996; 124(1 Pt 2): 86-89.

24 Effect of intensive blood-glucose control with metformin on complications in overweight patients with type 2 diabetes (UKPDS 34). UK Prospective Diabetes Study (UKPDS) Group. Lancet 1998; 352: 854-865.

25 Nathan DM, Cleary PA, Backlund JY, et al. Intensive diabetes treatment and cardiovascular disease in patients with type 1 diabetes. N Engl J Med 2005; 353: 2643-2653.

26 Holman RR, Paul SK, Bethel MA, et al. 10-year follow-up of intensive glucose control in type 2 diabetes. NEngl J Med 2008; 359: 1577-1589.

27 ADVANCE Collaborative Group; Patel A, MacMahon S, Chalmers J, et al. Intensive blood glucose control and vascular outcomes in patients with type 2 diabetes. NEngl J Med 2008; 358: 2560-2572.

28 Duckworth W, Abraira C, Moritz T, et al; VADT Investigators. Glucose control and vascular complications in veterans with type 2 diabetes. NEngl J Med 2009; 360: 129-139.

29 Action to Control Cardiovascular Risk in Diabetes Study Group; Gerstein HC, Miller ME, Byington RP, et al. Effects of intensive glucose lowering in type 2 diabetes. NEngl J Med 2008; 358: 2545-2559.

30 Cheung NW, Conn JJ, d'Emden MC, et al. Position statement of the Australian Diabetes Society: individualisation of glycated haemoglobin targets for adults with diabetes mellitus. Med J Aust 2009; 191: 339-344.

31 International Diabetes Federation Guideline Development Group. Guideline for management of postmeal glucose in diabetes. Diabetes Res Clin Pract 2014; 103: 256-268.

32 Balkau B, Hu G, Qiao Q, et al; DECODE Study Group; European Diabetes Epidemiology Group. Prediction of the risk of cardiovascular mortality using a score that includes glucose as a risk factor. The DECODE Study. Diabetologia 2004; 47 : 2118-2128.

33 Chiasson JL, Josse RG, Gomis R, et al; STOP-NIDDM Trial Research Group. Acarbose treatment and the risk of cardiovascular disease and hypertension in patients with impaired glucose tolerance: the STOP-NIDDM trial. JAMA 2003; 290: 486-494.

34 Colhoun HM, Betteridge DJ, Durrington PN, et al; CARDS investigators. Primary prevention of cardiovascular disease 
with atorvastatin in type 2 diabetes in the Collaborative Atorvastatin Diabetes Study (CARDS): multicentre randomised placebo-controlled trial. Lancet 2004; 364: 685-696.

35 Nadar S, Lim HS, Beevers DG, Lip GY. Lipid lowering in hypertension and heart protection: observations from the Anglo-Scandinavian Cardiac Outcomes Trial (ASCOT) and the Heart Protection Study. J Hum Hypertens 2002; 16: 815-817.

36 Basile J. Lessons learned from the ONTARGET and TRANSCEND trials. Curr Atheroscler Rep 2009; 11: 371-376.

37 Gaede P, Vedel P, Larsen N, et al. Multifactorial intervention and cardiovascular disease in patients with type 2 diabetes. $N$ Engl J Med 2003; 348: 383-393.

38 Royal Australian College of General Practitioners and Diabetes Australia. General practice management of type 2 diabetes - 2014-15. Melbourne: RACGP, 2014. http://www. racgp.org.au/your-practice/guidelines/diabetes (accessed Mar 2015).

39 Craig ME, Twigg SM, Donaghue KC, et al; Australian Type 1 Diabetes Guidelines Expert Advisory Group. National evidence-based clinical care guidelines for type 1 diabetes in children, adolescents and adults. Canberra: Australian Government Department of Health and Ageing, 2011. https:// www.nhmrc.gov.au/guidelines-publications/ext4 (accessed Mar 2015).

40 Knight AW, Ford D, Audehm R, et al. The Australian Primary Care Collaboratives Program: improving diabetes care. BMJ Qual Saf 2012; 21: 956-963.

41 Knight AW, Caesar C, Ford D, et al. Improving primary care in Australia through the Australian Primary Care Collaboratives Program: a quality improvement report. BMJ Qual Saf 2012; 21: 948-955.

42 Colagiuri S, Dickinson S, Girgis S, Colagiuri R. National evidence based guideline for blood glucose control in type 2 diabetes. Canberra: Diabetes Australia and National Health and Medical Research Council, 2009. http://diabetesaustralia. com.au/PageFiles/763/Final\%20Blood\%20Glucose\%20 Control\%20Guideline\%20August\%202009\%20\%282\%29. pdf (accessed Feb 2015). 